\title{
Félix Arnaudin : Chants populaires de la Grande- Lande
}

Parc Régional Naturel des Landes de Gascogne/Éditions Confluences. 1995 et 1997

\section{Pierre Bec et Eliane Gauzit}

\section{(2) OpenEdition}

Journals

Édition électronique

URL : http://journals.openedition.org/ethnomusicologie/725

ISSN : 2235-7688

\section{Éditeur}

ADEM - Ateliers d'ethnomusicologie

\section{Édition imprimée}

Date de publication : 1 janvier 2001

Pagination : 231-236

ISBN : 2-8257-0723-6

ISSN : $1662-372 X$

\section{Référence électronique}

Pierre Bec et Eliane Gauzit, «Félix Arnaudin : Chants populaires de la Grande-Lande », Cahiers d'ethnomusicologie [En ligne], 13 | 2001, mis en ligne le 09 janvier 2012, consulté le 01 mai 2019. URL: http://journals.openedition.org/ethnomusicologie/725

Ce document a été généré automatiquement le 1 mai 2019.

Tous droits réservés 


\title{
Félix Arnaudin: Chants populaires de la Grande-Lande
}

Parc Régional Naturel des Landes de Gascogne/Éditions Confluences. 1995 et 1997

\author{
Pierre Bec et Eliane Gauzit
}

\section{RÉFÉRENCE}

Félix Arnaudin : Chants populaires de la Grande-Lande. Édition établie et présentée par Jacques Boisgontier et Lothaire Mabru, 2 volumes. Parc Régional Naturel des Landes de Gascogne / Éditions Confluences. 1995. LXX + 452 p. / 1997. XIV + 837 p.

Ces deux recueils forment les volumes III et IV de l'ensemble des huit volumes des œuvres complètes de Félix Arnaudin, dont le Parc Régional des Landes de Gascogne a entrepris l'édition. Au magnifique album de photos (quelque trois mille plaques de verre), aux Contes (I), aux Proverbes, Dictons, Devinettes, Formulettes, Prières (II), viennent donc s'ajouter ces deux recueils de chants. Doivent suivre le Dictionnaire de la Grande-Lande, également en deux volumes, dont la parution a été retardée par le décès prématuré de Jacques Boisgontier, et enfin la Correspondance et les Écrits divers. Ensemble énorme que Félix Arnaudin (1844-1921) collecta systématiquement à partir de 1878 et pour lequel il fut poussé, suivant les matières étudiées, à faire un véritable travail de linguiste, de photographe, d'historien, d'ethnologue, de folkloriste, de chercheur... En effet, c'est à partir de cette date que, ayant déjà renoncé à exercer un métier et vivant du revenu de quelques métairies, il entreprenait sa collecte comme une sorte d'obligation personnelle : il se devait de noter les transformations de la vie sociale, du paysage, des coutumes qui survenaient à ses yeux avec l'introduction de la forêt industrielle, et qu'en observateur romantique, il déplorait. Engagé dans son entreprise de sauvegarde, il sillonne en bicyclette la Grande Lande (au total près de 20000 kilomètres, nous signale-t-on) et en chaque matière, il tient des cahiers, des registres, des répertoires d'une grande 
exhausivité: "il faudra encore de nombreuses années, nous dit-on, avant que les chercheurs d'aujourd'hui aient pu épuiser la richesse de ce travail !»

2 En fin du volume I des Chants, Guy Latry, chargé de l'édition de la correspondance de Félix Arnaudin, note que celui-ci travailla pendant dix ans à la préparation de son recueil : « un vrai travail de patience dont la fin semble reculer à mesure que j'en approche». De multiples brouillons de la préface montrent l'importance que ce recueil de chants revêtait pour lui, qui est plus un «investissement personnel... qu'un travail de spécialiste». "Ouvert par une préface, poursuit Guy Latry, où deux parties plus techniques sont encadrées par des textes d'une ampleur lyrique qui posent le véritable objet du livre (audelà des chants, l'ancienne Lande), clos par une chanson des communes qui parcourt la lande autour de Labouheyre..., le recueil dans son inachèvement proclamé («tome I»), apparait comme le substitut d'un autre ouvrage, ce « livre de la lande », « idée secrète de ma jeunesse », dont Arnaudin évoque à la fin de la préface le rêve inassouvi ».

$\mathrm{Du}$ vivant d'Arnaudin, seules trois publications parurent, dont un volume de Chants populaires (tome I, 1912); mais ces 196 chants «souvent enrichis de variantes... ne constituaient qu'une faible part des œuvres collectées pendant plus de trente années auprès de quelque 340 chanteurs ». Les manuscrits du reste de la collecte semblaient perdus ; cependant, on en découvrit une partie (84 chansons) en 1963, ce qui donna lieu à la parution d'un deuxième tome (1970) par Simone Wallon, Adrien Dupin et Jacques Boisgontier. Enfin, 201 autres inédits furent retrouvés, ce qui fait un nombre total de 481 chansons, auquel il faut ajouter de nombreuses variantes.

4 La présentation actuelle de ces deux tomes de chants est fort belle, voire même luxueuse, agrémentée de reproductions de pages manuscrites, de photos prises par Arnaudin et de deux portraits-photos de lui-même.

5 Le volume I reprend l'édition de 1912 et fournit des Chants du premier âge (berceuses amusettes) et des Chansons de danse (rondes enfantines - chansons de neuf - chansons énumératives - chansons facétieuses et burlesques - chansons satiriques). On y a ajouté, au début du livre, une introduction de Lothaire Mabru (cf. ci-dessous) et, en annexes à la fin - initiative très utile - des comptes rendus critiques publiés par la presse de l'époque et des inédits d'Arnaudin sur les instruments de musique. Ces inédits, documents bruts, se présentent sous forme d'enquêtes, c'est-à-dire que pour chaque instrument, Arnaudin donne le nom de la personne interrogée, son âge, son lieu d'habitation, le jour de l'enquête et la réponse de cette personne en gascon à une ou des question(s) que nous ne connaissons pas, mais qui se laisse(nt) facilement deviner. Tour à tour sont présentés deux sortes de hautbois, trois sortes de trompes de bois, la cornemuse, le fifre, la guimbarde, la vielle, le violon, le tambourin et l'accordéon.

6 Le tome II, après une autre introduction de L. Mabru, reprend les 70 chants déjà présentés dans l'édition de S. Wallon et J. Boisgontier, avec quelques modifications (cf. plus loin) et y ajoute tous les autres inédits. On y trouve la suite des Chansons de danse (chansons d'amour, chansons anecdotiques et légendaires) et des Chants divers (chansons à voix - chants de moissonneurs - complaintes - chants religieux - chants cérémoniels - chants de charivaris et d'" asouades » - chansons nouvelles et étrangères) et un supplément aux chansons de ronde.

7 Le travail de révision, de dépouillement et de déchiffrage des manuscrits a été confié à deux spécialistes: Jacques Boisgontier pour les paroles et Lothaire Mabru pour la musique. 
8 Jacques Boisgontier, gascon, spécialiste de dialectologie romane, a vécu pendant de longues années dans une quasi intimité avec les écrits d'Arnaudin. Ayant déjà participé à l'établissement des paroles des premières éditions ou rééditions (Proverbes, paru en 1965 ; Contes en 1967 et 1994 ; tome II des Chansons en 1970), il travailla avec acharnement et minutie pour l'élaboration de cette nouvelle édition. On peut se rendre compte de la tâche accomplie en examinant par exemple les quelques photos de "minutes" reproduites surtout dans le tome II des Chants, ou en lisant son texte court et précis, Graphie et lecture du gascon, paru dans le volume des Contes. Besogne qui peut aller jusqu'à la minutie la plus scrupuleuse - voir, par exemple ses remarques intercalées dans le chapitre d'Arnaudin "De la prononciation", au début du tome I des Chants. De plus, Jacques Boisgontier, spécialiste entre autre du gascon et plus particulièrement de sa variété dialectale, le grand-landais, était le chercheur privilégié pour accomplir cette tâche. Le résultat de cette magnifique édition ne rend pas compte - car Jacques était modeste - de la somme de connaissances accumulées nécessaires à une telle élaboration et à une maîtrise si sentie de la langue. De plus, il est bon de signaler qu'il a participé à de très nombreuses enquêtes sur le terrain pour la préparation des Atlas linguistiques et ethnographiques du Languedoc - ce qui lui a permis en outre d'amasser des documents sonores fort intéressants (contes, chants, témoignages ethnographiques, etc.) - et qu'il a largement collaboré à leur rédaction.

Lothaire Mabru, docteur en anthropologie sociale et ethnologue, fut chargé de la relecture de la musique pour le tome I de ces Chansons et de son établissement pour le tome II. Par ses enquêtes sur les pratiques musicales en milieu rural (par exemple, sur la cornemuse des Landes de Gascogne) et ses différents travaux de recherche, il était tout indiqué pour participer à ce travail et, en particulier, pour commenter les connaissances organologiques d'Arnaudin (Introduction du T. I, p. X à XV). Dans cette même introduction, il insiste à plusieurs reprises sur le fait que, tant au niveau du texte que de la musique, l'examen des manuscrits permet d'affirmer qu'Arnaudin, dans ce tome I qu'il fit imprimer, donna comme versions définitives des textes reconstitués. Et il explique cette attitude par le fait que c'est la méthode employée par les folkloristes de l'époque: " leur travail consistait à opérer parmi les matériaux une sélection, selon des critères esthétiques personnels et forcément arbitraires : ne retenir, pour la musique, que les mélodies les plus caractéristiques et, pour les textes établir une version moyenne à partir de toutes les dictées individuelles recueillies » (J. Boisgontier, Chants II, 1970). D'autre part, Mabru souligne qu'il importait à Arnaudin non de faire une compilation de chants , mais de présenter par les chansons une vision la plus exacte et complète possible de « son » ancienne lande.

Aussi avons-nous envie de poser une question à propos de ce volume I : puisque les manuscrits retrouvés de ce premier tome donnent plus ou moins les textes bruts utilisés par Arnaudin, pourquoi ne pas envisager, dans la mesure du possible, d'en prévoir l'édition?

11 Et cette question nous amène au tome II. En effet, L. Mabru souligne que, grâce aux minutes d'enquêtes, « les chants publiés ici représentent des productions individuelles de chanteurs dont l'identité est précisée ». D'où cette récolte d'une richesse exceptionnelle : dans de nombreux cas, la même chanson est présentée, tant pour les paroles que pour la musique, sous 3, 4 et même 5 occurrences divergentes. Ces chants peuvent être classés, pour la plupart, dans la grande production du territoire hexagonal de l'État français, élargi de la Catalogne et du Piémont (cf. les Catalogues de Conrad Laforte et ceux de 
Patrice Coirault). La grande originalité semble surtout se trouver dans certaines mélodies qui pour nos oreilles sonneraient étrangement, ainsi que le souligne Lothaire Mabru. Personnellement, j'ai été charmé par des pièces courtes classées dans les Chants divers. Par exemple cette cante de sega [chant de moisson] à la langue savoureuse soutenue par une mélodie modale :

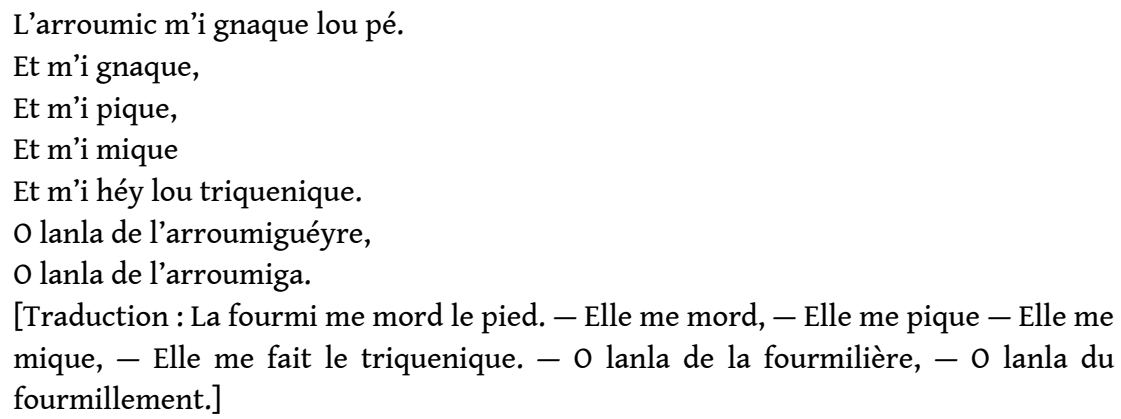

Pour ce deuxième tome, Mabru précise qu'il convient de distinguer deux ensembles. Le premier correspond à l'édition de 1970 (p. 1 à 327) et il explique pourquoi il y a certaines divergences entre sa transcription et celle de $\mathrm{S}$. Wallon. Pour le deuxième ensemble ( $\mathrm{p}$. 328 à 810 ) regroupant les chants restés inédits jusqu'à ce jour, Mabru déclare que son établissement «a posé de nombreux problèmes qu'il a bien fallu résoudre »; Et il ajoute : «je tiens à redire que mon souci constant a été de m'en tenir au plus près des manuscrits ». Il est vraiment regrettable que, dans cette splendide édition, la rigueur scientifique observée par Jacques Boisgontier pour la transcription des paroles ne se retrouve pas chez Lothaire Mabru pour la transcription de la musique et des paroles sous la musique.

13 En effet, il semblerait qu'aucune méthodologie bien assurée ne l'ait guidé. Nous signalerons rapidement quelques oublis ou erreurs d'écriture dans la musique. A plusieurs reprises il manque la double barre de mesure en fin de partition (ex. p. 65) ; des croches pointées - temps binaires - ne sont pas suivies de doubles croches (ex. p. 346, 2ème temps) ; des vers sont bissés, mais il n'y a pas d'indication de reprise de la musique (ex. p. 587). On peut se demander pourquoi certaines partitions musicales ne comportent pas de paroles. L.M. dit dans l'introduction: "lorsque les manuscrits donnent des partitions sans les paroles, j'ai fait de même». Or, dans les reproductions de certaines minutes, Arnaudin ne met pas les paroles sous la musique (ex. p. 677 ou 695); mais les transcriptions notent cependant ces paroles (p. 100 et 102). Il semblerait qu'Arnaudin n'ait pas toujours mis les paroles sous la musique, quand il n'y avait aucune équivoque concernant leur concordance. Cette même chanson p. 100 devrait avoir un la bémol à la mesure 3, bémol bien visible dans le manuscrit d'Arnaudin. Ce manque de logique se retrouve dans la manière de noter les vocalises, en général de deux notes : au début du livre un signe de liaison, souvent mis à l'envers, unit les deux notes, plus loin on trouve des croches assemblées en plus du signe de liaison, puis, à partir de la p. 328, on revient au système du début.

14 Ce même illogisme se retrouve dans le découpage des mots en syllabes et leur mise en place sous les notes. En particulier, dans de nombreux cas, il n'est pas tenu compte des synalèphes (voyelle finale d'un mot prononcée avec la voyelle débutant le mot suivant) qui sont nombreuses en occitan, et sans doute plus encore en gascon landais : une lecture attentive des paroles et un déchiffrage de la chanson auraient peut-être permis d' éviter de nombreuses erreurs. Par exemple p.126, les quatre pieds se découpent de la façon 
suivante : $\underline{b o}-\underline{n e} a r-\underline{r i}-\underline{b e}$ (nous soulignons chaque pied) et plus loin dans la même chanson on doit avoir : $\underline{a r}-\underline{r i}-\underline{b e} a r-\underline{r}-\underline{b e}$; ce que L. M. découpe ainsi : $\underline{b o n e} \underline{a r}-\underline{r} \underline{-}-\underline{b e}$

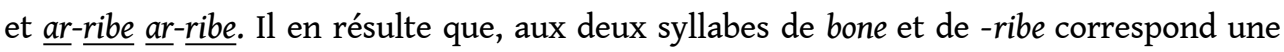
seule note ( de même pour l'amou p. 448) et que le même mot arribe, qui est répété, est découpé par L. M. en trois puis en deux syllabes! A d'autres endroits, la structure même de la langue est dénaturée: ainsi p. 804, minj'rî est écrit min-j'ri; ou encore une diphtongue ou un mot d'une syllabe sont coupées en deux (ex. p. 391, riu-le écrit ri-ule et p. 396 lugn écrit lu-gn !! !). Enfin en de nombreux endroits la dernière syllabe des mots se terminant par un -e atone est omise, comme anade (écrit a-nade), de même qu'une quantité d'onomatopées coupées ainsi : lan-lire, don-dène, de-ri-dète, etc.).

A plusieurs reprises, le non-respect des vocalises et du découpage des mots en syllabes entrainent dans certains cas des fautes graves dans le rapport musique/paroles et dénaturent complètement les chansons en question. En règle générale, les paroles bissées sont chantées sur le même air ou un air semblable, et il en est de même pour les refrains qui sont le plus souvent chez Arnaudin des onomatopées. Prenons l'exemple de la chansons XC bis, p. 422, L'aute journ en m'i permenan. Cette phrase bissée devrait être rechantée en recommençant sur le la ( $2^{\mathrm{e}}$ ligne, $1^{\mathrm{re}}$ mesure) : on aurait comme cela deux phrases musicales semblables, la ré mi ré ré la ré do si. Il semblerait que L. M. n'ait pas pris en compte une vocalise sur le mot $m^{\prime} i$ (notes ré la, troisième mesure), et pour cela il faudrait vérifier sur le manuscrit, car Félix Arnaudin marque les notes liées sans aucune équivoque. (Cf. par ex. le cliché p. VIII et sa transcription p. 65 : on y retrouve le même incipit avec une vocalise sur $\left.m^{\prime} i\right)$. Cette première phrase musicale notée ainsi avec un liaison permet de faire coïncider l'accent métrique de permenan qui est sur -nan avec l'accent musical. La solution proposée par L. M. est anti-musicale et il faut faire un très gros effort pour la chanter. Le deuxièmeme vers se déroule très facilement: à chaque syllabe correspond une note et il ne faut pas tenir compte des liaisons qui semblent avoir été ajoutées par L. M. (vérifier sur le mss); de cette façon cela enlèverait la syncope de l'avant-dernière mesure, syncope qui n'est pas dans le style de cette musique. Et il en est de même pour de nombreuses chansons. Nous avons noté que, dans la partie qui est commune avec l'édition de 1970, il y a quatre chansons transcrites avec des fautes graves et qu' à partir de la p. 328 il y en a quarante-sept... dont certaines sont de véritables «monstres » anti-musicaux (phrases ou mots coupés par un silence : ex. p. 388, p. 567 ; les paroles s'arrêtent alors que la mélodie continue : ex. p. 198, dernière mesure; mot ajouté pour que « ça colle »; ex. p. 506, onomatopée la ajoutée, à la répétition des deux premiers vers). Enfin plusieurs fois, L. M. dit qu'il n'a pas pu faire de transcription musicale, parce qu'il y a soit trop de notes, soit trop de paroles et pourtant, il nous semble qu'il y avait des solutions possibles (cf. par ex. p. 394).

En résumé, on peut se poser la question de savoir pourquoi Lothaire Mabru, qui a fait ailleurs preuve de solides qualités de chercheur, a présenté dans ce deuxième tome un travail défectueux tant pour la connaissance du gascon - on sait qu'il n'a guère tenu compte de la collaboration de Jacques Boisgontier - que pour la transcription musicale.

Cette indispensable édition des œuvres complètes de Félix Arnaudin aurait mérité dans sa transcription musicale plus de minutie, plus de scrupules dans la transmission du manuscrit original, voire plus de respect pour la cause défendue. 